\title{
A MAP-BASED APPROACH TO RESOLUTION ENHANCEMENT OF HYPERSPECTRAL IMAGES
}

\author{
Hasan IRMAK ${ }^{1,2}$, Gözde B. AKAR ${ }^{2}$, Seniha Esen YÜKSEL ${ }^{3}$ \\ (1) Aselsan Inc, Turkey \\ (2) Middle East Technical University, Dept. of Electrical and Electronics Eng., Turkey \\ (3) Hacettepe University, Dept. of Electrical and Electronics Eng., Turkey
}

\begin{abstract}
Hyperspectral imaging is widely used in many fields such as geology, medicine, meteorology, and so on. Despite the high spectral resolution, the spatial resolution of the hyperspectral sensors is severely limited. In this paper, we propose a novel maximum a posteriori (MAP)-based approach based on the joint superresolution of the abundance maps, to enhance the resolution of hyperspectral images. In the proposed approach, first, the endmembers and their abundance maps are estimated using Vertex Component Analysis (VCA) and Fully Constrained Least Squares (FCLS), respectively. Second, a high resolution (HR) abundance map is reconstructed for each low resolution (LR) abundance map using a MAP-based approach. In the MAP-formulation data, smoothness and edge preservation constraints are extended to include a unity constraint term specific to abundances. The proposed algorithm is tested on both synthetic images and real image sequences. The experimental results and comparative analysis verify the effectiveness of the proposed algorithm.
\end{abstract}

Index Terms - Hyperspectral, Super-resolution, Spectral Unmixing, Markov Random Field, Graph Cut Energy Minimization

\section{INTRODUCTION}

Hyperspectral sensors have the ability to acquire images in many narrow spectral bands in the electromagnetic spectrum. Despite the high spectral resolution, the spatial resolution of the hyperspectral sensors is severely limited because of the limitations of the imaging hardware [1]. The limit of resolution can lead to mixed pixels that are pixels occupied by two or more pure elements, called endmembers. These mixed pixels adversely affect the detection and recognition performance in many practical applications. In the literature, many algorithms are proposed to increase the resolution of hyperspectral images [1][2][3]. Several researchers have studied spectral mixture analysis to find subpixel information [3][4]. These studies estimate the abundance maps and obtain super-resolution land cover maps by finding subpixel locations of the endmembers.
Generally, interpolated abundances are assumed to consist of pure pixels which may not be an accurate assumption [5]. Another approach is using super-resolution mapping by fusing a hyperspectral image with a High Resolution (HR) image [6]. However, in general, obtaining a hyperspectral image with a HR panchromatic or multispectral image is difficult. There are also neural network based methods which require a learning phase [7]. In this paper, we propose a totally new approach where the abundance maps are jointly superresolved, to enhance the resolution of hyperspectral images. We treat each abundance map corresponding to an endmember as a separate source of information; and we use the subpixel information from LR abundance maps to reconstruct HR abundances. Experiments have been performed on both synthetic and real data to assess the effectiveness of the method. The remainder of the paper is organized as follows. Section II gives a detailed description of the proposed approach. Section III presents the experimental results and Section IV gives the conclusion and future work.

\section{MAP-BASED HYPERSPECTRAL SUPER- RESOLUTION}

The main idea is of the proposed approach is to jointly utilize the abundance fractions of the endmembers present in the scene in the resolution enhancement process. In our approach the abundance maps are superresolved as opposed to interpolating the spectra of each pixel. This approach needs to interpolate only a few abundance maps to construct the resolution enhanced hyperspectral image in all spectral bands. Therefore, it is also a time-efficient way of resolution enhancement for hyperspectral images. The block diagram of the proposed method is given in Figure 1. First, spectral unmixing is applied which is the combination of endmember extraction and abundance map estimation. After abundance maps are extracted, HR abundance maps are generated using MRF based energy model with Graph Cut optimization. Finally, abundance maps are merged to obtain HR image in each spectral band. 


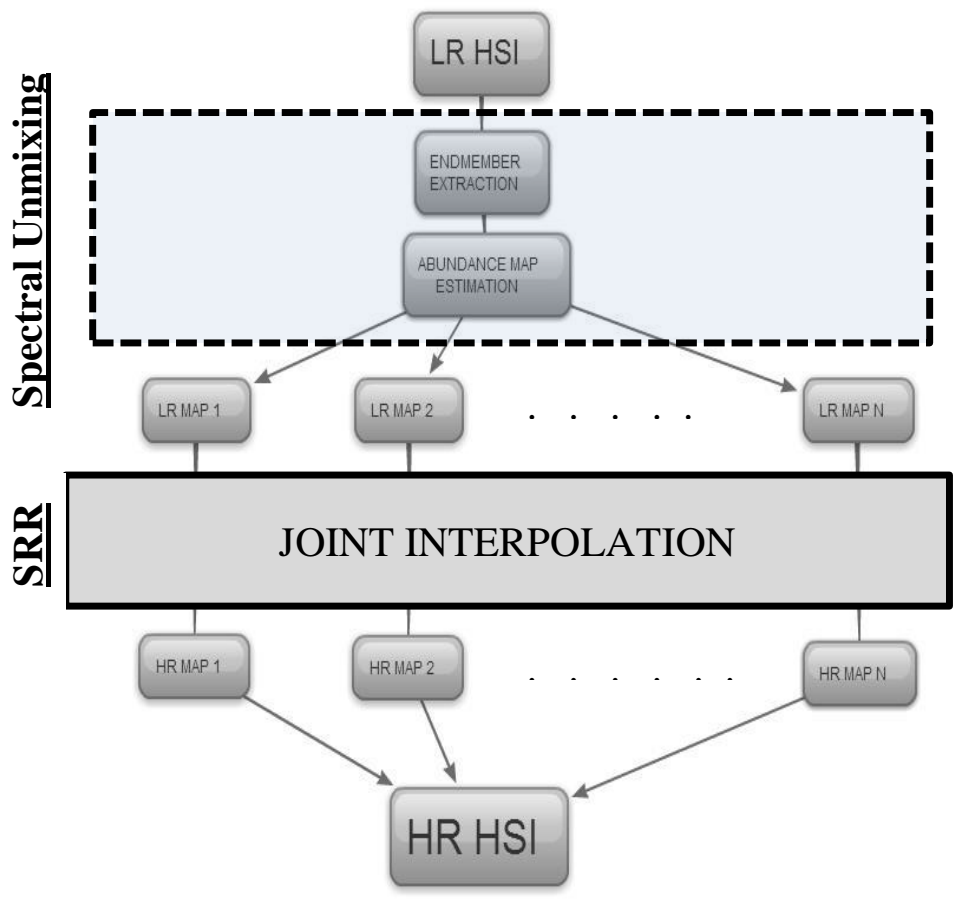

Figure-1: Block diagram of the proposed method. LR abundance maps of each endmember is interpolated through MRF and Graph cuts to obtain HR abundance maps, which are then combined to get a HR hyperspectral image.

\subsection{Spectral Unmixing}

In the linear spectral mixture model, any pixel in the scene can be expressed as a linear combination of the endmembers. The goal of spectral unmixing is to find the abundances of the endmembers in the image. In general, there are three main stages in spectral unmixing, namely dimension reduction (optional), endmember selection and abundance map estimation. There are various spectral unmixing algorithms in the literature [8]. In this study, we have selected the Vertex Component Analysis (VCA) [9] algorithm to find the endmembers in the scene for its fairly good performance and lack of need for dimension reduction. For the abundance map estimation, we have selected the Fully Constraint Least Squares (FCLS) which models the material quantification accurately enough [10].

\subsection{Super-resolution Reconstruction (SRR)}

The observation model that related the HR abundance maps to the LR abundance maps can be given as with the constraint of summation of the abundances for each pixel is unity. We use the following observation model:

$$
\mathrm{A}_{\mathrm{k}}=B \cdot D \cdot \tilde{\mathrm{A}}_{k}+n_{k}
$$

In this observation model, $\mathrm{A}_{\mathrm{k}}$ and $\tilde{\mathrm{A}}_{k}$ are the LR and HR abundance maps of the $\mathrm{k}^{\text {th }}$ endmember respectively and $\mathrm{n}_{\mathrm{k}}$ is the observation Gaussian noise. $B$ and $D$ are the blurring and downsampling operations. Moreover, in this model, summation of the LR abundance maps is unity:

$$
\sum_{k} A_{k}=1
$$

The problem of finding $\tilde{A}_{k}$ given $\mathrm{A}_{\mathrm{k}}$ is an ill-posed inverse problem and SRR algorithms can be used to find the HR abundance maps (i.e. $\tilde{A}_{k}$ ). Bayesian methods are effectively used in SRR problem [11]. These methods insert probabilistic information to the problem. In MAP-based approach, the most probable HR image is searched iteratively using the image prior knowledge. We use this model as a base model and extend the model with additional constraint terms related to abundance maps. Minimization of the energy functions for each endmember construct HR abundance maps. Merging these HR abundances gives us HR hyperspectral images in each spectral band.

\subsubsection{Energy Function}

In order to determine the energy function, we start from an initial energy model which is based on MAP-MRF framework defined as [12]:

$$
E=E_{d}+\lambda E_{s}
$$

The first term in this equation can be named as the data constraint that guarantees the consistency of the HR abundance maps with the observed data and the second as the a priori information. When we use MRF as the prior model, our goal is to obtain smooth HR abundance maps while keeping the original LR maps. Moreover, for the LR abundance maps, summation of the abundances for each pixel is unity, and this constraint should also hold for the HR abundance maps. Therefore, there is an interrelation between HR abundance maps. Finally, to preserve edges and prevent the over-smoothing, edge preserving regularization is required in the energy function. Combining all these constraints, we define an energy function that has data, edge preserving and smoothness terms in order to meet the requirements of the prior model. These terms are explained below:

1) Data Constraint (DC): Assuming the model noise is AWGN, the data constraint term can be written as 


$$
D C=\sum_{j=1}^{N}\|D \cdot B \cdot(S . \tilde{\mathrm{A}})-S \cdot A\|
$$

where $\quad D$ : Downsampling Operation

$B$ : Blurring Operation

$S$ : Spectral Signatures of Endmembers

Ã: HR Abundance Map

A: LR Abundance Map

$N$ : Number of Pixels

2) Smoothness Constraint (SC): Arrangement should reflect a spatial consistency in HR abundance map.

$$
S C=\sum_{k=1}^{N} \sum_{j=1}^{4}\left\|\tilde{\mathrm{a}}(k)-\tilde{\mathrm{a}}_{\text {clique }}(k)(j)\right\|
$$

where $\tilde{a}(\mathrm{k})$ is the abundance of pixel $\mathrm{k}$; and $\tilde{\mathrm{a}}_{\text {clique }}$ is the 4neighborhood of $\tilde{a}(\mathrm{k})$.

3) Unity Constraint (UC): In HR Image, sum of the abundances of a single pixel should be unity.

$$
\sum_{e=1}^{E}\|\tilde{\mathrm{a}}(k)(e)\|=1 \text { for } \forall k
$$

where $E$ is the number of endmembers.

4) Edge Preserving Constraint (EPC): In HR Image, edges of the LR images should be preserved if gradient is greater than a threshold. In other words, we masked this constraint using mask coefficients $(M x, M y)$ if gradients $(G x, G y)$ are smaller than a threshold:

$$
\begin{aligned}
& M x(i, j)=1 \text { iff } G x(i, j) \geq \text { Threshold, otherwise } 0 \\
& M y(i, j)=1 \text { iff } G y(i, j) \geq \text { Threshold, otherwise } 0
\end{aligned}
$$

Using the mask coefficients, we can define the EPCs in $\mathrm{x}$ and y directions:

$$
\begin{aligned}
& \operatorname{EPCx}(i, j)=M x \cdot\|(\tilde{\mathrm{A}}(i, j)-\tilde{\mathrm{A}}((i+1), j))\| \\
& \operatorname{EPCy}(i, j)=M y \cdot\|(\tilde{\mathrm{A}}(i, j)-\tilde{\mathrm{A}}(i,(j+1)))\|
\end{aligned}
$$

Sum of the EPCs in both directions gives us the total EPC:

$$
E P C=\sum_{i=1}^{R_{H}} \sum_{j=1}^{C_{H}}\|E P C x(i, j)+\operatorname{EPCy}(i, j)\|
$$

where $R^{H}$ is the number of rows in the HR abundance map and $C^{H}$ is the number of columns in HR abundance map.

Combining the terms related to data constraint and a-priori information, the energy function that is to be minimized can be written as:

$$
E=\alpha \cdot D C+\beta \cdot S C+\gamma \cdot E P C
$$

Minimization of the energy function $\mathrm{E}$ for each endmember while satisfying the UC condition gives the HR abundance maps. DC, SC and EPC are independent for each endmember whereas UC is a joint constraint between endmembers. Therefore, HR abundance maps are jointly checked for the UC condition.

\subsubsection{Minimization of Energy Function}

In [12], different energy minimization methods such as Iterated Conditional Modes (ICM), Graph Cut Expansion (GCE), Graph Cut Swap (GCS), Max-Product Loopy Belief Propagation (LBP) are compared and benchmarks are given. In these benchmarks, it is concluded that GCE is clearly the winner in terms of runtime. Moreover, it performs best minimization in energy in majority of the cases. Therefore, we selected GCE as the Energy minimization method for our study. GCE algorithm searches a labeling such that no expansion move for any label $\alpha$ yields a labeling with lower energy [13]. Although, Graph Cut is typically used for labelling problems, recently it is also being used for the SRR problem [14] We treat each different abundance of an endmember as a label and apply GCE method to enhance the resolution by finding the optimal labeling by minimizing the given energy function.

\section{EXPERIMENTAL RESULTS}

We applied the proposed method to both synthetic and real hyperspectral images. Due to page limit, we only give the results of real hyperspectral image experiments. Image dataset consists of 31 narrow bands, each with approximately $10 \mathrm{~nm}$ bandwidth and centered at steps of $10 \mathrm{~nm}$ from $420 \mathrm{~nm}$ to $720 \mathrm{~nm}$ [15]. Instead of the whole image, 160x160 patches are used in the experiments. 80x80 input test images are generated applying $2 x$ downsampling to these image patches. These test images are input to the proposed system. The output is the $2 \mathrm{x}$ resolution enhanced version (160x160) of the 80x80 input test images. Result of the proposed method is compared with original $160 \times 160$ image, bicubic interpolated image and graph cut interpolated image. Bicubic and graph cut interpolation is directly applied to the downsampled hyperspectral image as in proposed method. The results of the hyperspectral image are shown in Figure 2 and 3 for the first spectral band (i.e. 420 
nm). Visually, the proposed method is promising as compared to other interpolated images. Bicubic interpolated image is degraded by smoothing. Graph Cut Interpolated image is adversely affected from the noise. However, our proposed method preserves the edges and filters the noise in the hyperspectral image. In the experiments, we use $\{\alpha=5$, $\beta=2, \gamma=100\}$ coefficients as the weights of the constraints of energy function $\mathrm{E}$.

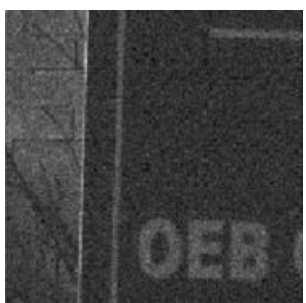

(a)

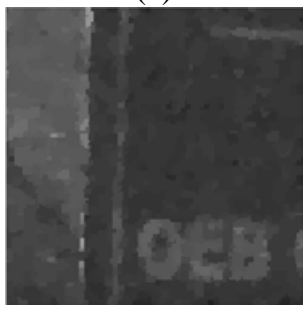

(c)

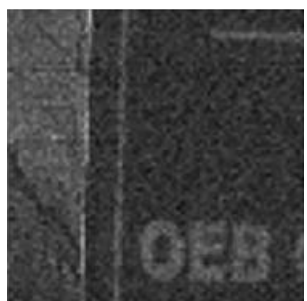

(b)

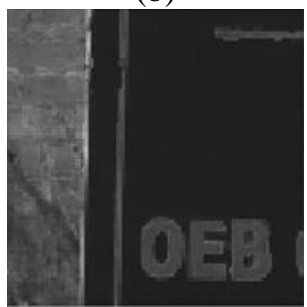

(d)
Figure 2: Comparison of the super-resolution methods on one band. (a) Original Image, (b) Bicubic Interpolated Image, (c) Graph Cut Interpolated Image, (d) Proposed Method.

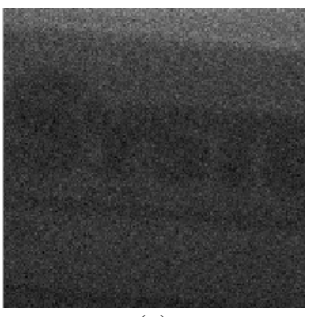

(a)

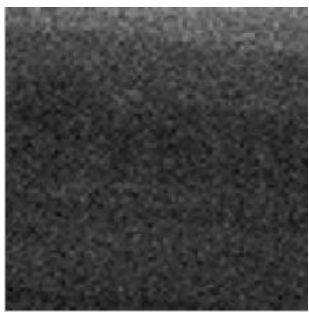

(c)

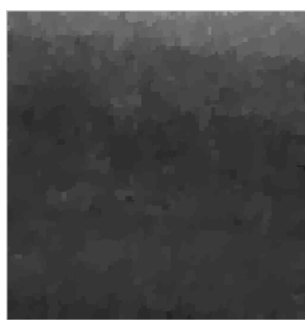

(b)

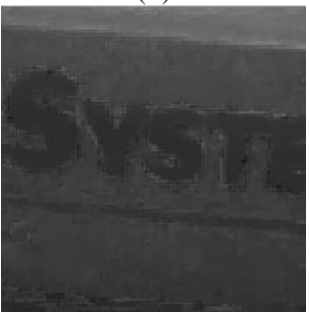

(d)
Figure 3: Comparison of the super-resolution methods on one band. (a) Original Image, (b) Bicubic Interpolated Image, (c) Graph Cut Interpolated Image, (d) Proposed Method.

\section{CONCLUSIONS}

This paper presented a spectral unmixing based approach for the super-resolution of hyperspectral images. The proposed method is tested on both synthetic and real hyperspectral images which have promising results. Further experiments will be conducted in order to quantitatively evaluate the performance of the proposed method.

\section{REFERENCES}

[1] H. Zhanga, L. Zhanga and H.Shen, "A super-resolution reconstruction algorithm for hyperspectral images" Signal Processing 92 pp 2082-2096, 2012.

[2] T. Akgün, Y. Altunbaşak ve R. M. Mersereau, "Superresolution reconstruction of hyperspectral images", IEEE Transactions on Image Processing, vol. 14, issue 11, pp. 18601875, November 2005.

1. [3] T. Kasetkasem,M.K.Arora, and K.Varshney, "Superresolution land cover mapping using a Markov random field based approach", Remote Sensing of Environment, 96, pp302- 314,2005

2. [4] A. Villa, J. Chanussot, J. Benediktsson, M. Ulfarsson, C. Jutten, "Super-resolution: an efficient method to improve spatial resolution of hyperspectral images", IGARSS, 2010.

[5] G. Martin, J.Plaza, and A. Plaza, "On the incorporation of spatial information to endmember identification algorithms without the pure pixel assumption" Hyperspectral Image and Signal Processing: Evolution in Remote Sensing (WHISPERS), pages 1-4, 2011.

[6] Y.Zhao, Y.Yang, Q.Zhang, J.Yang, "Hyperspectral imagery super-resolution by image fusion and compressed sensing", IGARSS, 2012.

[7] F.A. Mianji , Y.Zhang, Y. Gu, "Resolution Enhancement Of Hyperspectral Images Using A Learning-Based Super-Resolution Mapping Technique”, IGARSS, 2009.

[8] N.Keshava, "A survey of spectral unmixing problem", Lincoln Laboratory journal, vol.14, no. 1, 2003.

[9] I.Gerg, "An Evaluation Of Three Endmember Extraction Algorithms: Atgp, Ica-Eea \& Vca", IEEE Trans., 2010.

[10]D. C. Heinz, C. Chang, "Fully Constrained Least Squares Linear Spectral Mixture Analysis Method for Material Quantification in Hyperspectral Imagery", IEEE Transactions on Geoscience and Remote Sensing, Vol. 39, No. 3, March 2001

[11] L. C. Pickup, D. P. Capel, S. J. Roberts, A. Zisserman, "Bayesian Methods for Image Super-resolution", The Computer Journal, 2007.

[12] R. Szeliski, R. Zabih, D. Scharstein, "A Comparative Study of Energy Minimization Methods for Markov Random Fields with Smoothness-Based Priors", IEEE Transactions on Pattern Analysis and Machine Intelligence, Vol. 30, No. 6, June 2008.

[13] Y. Boykov, O. Veksler, and R. Zabih, "Fast Approximate Energy Minimization via Graph Cuts", IEEE Transactions, 2001.

[14] U. Mudenagudi, R. Singla, P. K. Kalra, and S. Banerjee. "Super-resolution using graph-cut", ACCV, pp.385-394, 2006

[15] A. Chakrabarti and T. Zickler, "Statistics of Real-World Hyperspectral Images", IEEE Conference on Computer Vision and Pattern Recognition (CVPR), 2011. 